\title{
Cohomological Vertex Operators
}

\author{
ANDRÉS VIÑA \\ Departamento de Física, Universidad de Oviedo, Avda Calvo Sotelo, 33007 Oviedo, Spain \\ Email:vina@uniovi.es
}

\begin{abstract}
Given a Calabi-Yau manifold and considering the $B$-branes on it as objects in the derived category of coherent sheaves, we identify the vertex operators for strings between two branes with elements of the cohomology groups of Ext sheaves. We define the correlation functions for these general vertex operators. Strings stretching between two coherent sheaves are studied as homological extensions of the corresponding branes. In this context, we relate strings between different pairs of branes when there are maps between these pairs. We also interpret some strings with ghost number $k$ as obstructions for lifts or extensions of strings with ghost number $k-1$.
\end{abstract}

Keywords: $B$-branes, vertex operators, derived categories of sheaves

\section{Introduction}

In the nonlinear sigma $B$-model on a Calabi-Yau manifold $X$, the local operators are defined by differential forms of type $(0, q)$ with coefficients in the vector bundles $\bigwedge^{p} T X$, where $T X$ is the holomorphic tangent bundle of $X$ [21]. The BRST operator in this theory is the Dolbeault operator $\bar{\partial}$; so, the spaces $H_{\bar{\partial}}^{q}\left(X, \bigwedge^{p} T X\right)$ are the groups of the BRST cohomology of the $B$-model.

The coupling of that system to gauge fields is carried out through Chan-Paton factors [20]. In this way, given two holomorphic vector bundles $V_{1} \rightarrow X$ and $V_{2} \rightarrow X$, an open string vertex operator for a string stretching from $V_{1}$ to $V_{2}$ is given by a $\bar{\partial}$-closed $(0, q)$-form with values in the vector bundle $\operatorname{Hom}\left(V_{1}, V_{2}\right)$. More precisely, the vertex operators for strings between the $B$-branes defined by $V_{1}$ and $V_{2}$ are the elements of the cohomology groups (see [1, Sect. 3.2.2], [2, page 207])

$$
H_{\bar{\partial}}^{0, q}\left(X, \operatorname{Hom}\left(V_{1}, V_{2}\right)\right) .
$$

In this note, we will deal with $D$-branes of type $B$ in a compact $n$-dimensional Kähler manifold $X$ and with other objects related to them. Such a brane can be considered as an object of $D(X)$, the bounded derived category of coherent sheaves on $X$ (see monograph [2], which includes an exhaustive list of specific references). Particular $B$-branes are the holomorphic vector bundles on $X$, and (1) gives the spaces of local operators for strings stretching between two of these particular branes.

Obviously, (1) makes non sense for strings between general branes. One of the purposes of this article is to "extend" that formula to the case of strings stretching between two arbitrary branes; that is, to give a general definition of the spaces of vertex operators which coincides with (1) when the branes are locally free sheaves.

In Section 2, we carry out the mentioned "extension". Firstly, we recall the identification of the space (1) with the $q$ th group of cohomology of $X$ with coefficients in the sheaf of holomorphic sections of $\operatorname{Hom}\left(V_{1}, V_{2}\right)$. On the other hand, if $\mathcal{F}$ and $\mathcal{G}$ are $B$-branes, i.e., objects of the category $D(X)$, an open string between $\mathcal{F}$ and $\mathcal{G}$ with ghost number $k$ is an element of the Ext group $\operatorname{Ext} t^{k}(\mathcal{F}, \mathcal{G})$ [1, Sect. 5.2]. As $H o m=E x t^{0}$, the strings in $\operatorname{Hom}\left(V_{1}, V_{2}\right)$ have ghost number 0 . So, the vertex operators considered in the space (1) are for strings with zero ghost number, and we do not have a definition of local operators for strings between general branes with arbitrary ghost number.

When one considers two general branes $\mathcal{F}$ and $\mathcal{G}$ and strings with ghost number $k$, as a direct generalization of formula (1), we propose

$$
\bigoplus_{q} H^{q}\left(X, \mathcal{E} x t^{k}(\mathcal{F}, \mathcal{G})\right),
$$


for the corresponding space of vertex operators (see (13)). In this way, the vertex operators are regarded as elements of sheaf cohomology groups.

These spaces of vertex operators for strings from a locally free sheaf $\mathcal{E}$ to a coherent sheaf $\mathcal{G}$ are specially simple: the local operators corresponding to strings with ghost number $k>0$ are trivial (Proposition 2.5). Moreover, the strings with ghost number $k$ from $\mathcal{E}$ to $\mathcal{G}$ can be identified with vertex operators for strings with ghost number 0 stretching from $\mathcal{E}$ to $\mathcal{G}$ (Proposition 2.6).

When $X$ is a Calabi-Yau manifold of dimension $n$, there is a holomorphic volume form which permits to identify the space $\bigwedge^{n} T X$ with the space of holomorphic $n$-forms. Thus, in the sigma $B$-model, the correlation functions corresponding to local operators can be calculated by integration on $X$ of corresponding differential forms.

In our case, when $X$ is a projective Calabi-Yau variety, using the Serre duality in the derived category $D(X)$, the holomorphic volume form and the Yoneda product, we will define the correlation functions for the local operators introduced above (see (16)). In Proposition 2.9, we prove that this definition generalizes the one given in [2, page 208] for operators of strings between holomorphic vector bundles.

We denote with $\mathfrak{C o h}(X)$ the category of coherent sheaves on $X$. The category $D(X)$, as derived category of $\mathfrak{C o h}(X)$, is triangulated [13, Sect 1.5]. Hence, we can consider distinguished triangles in $D(X)$

$$
\mathcal{B} \stackrel{u}{\rightarrow} \mathcal{C} \stackrel{v}{\rightarrow} \mathcal{D} \stackrel{+1}{\rightarrow} \mathcal{B}[1]
$$

In physical terms, this triangle can be interpreted as a possible binding of the branes $\mathcal{B}$ and $\mathcal{D}$ to form the brane $\mathcal{C}$ [2, page 368]. In general, a string from $\mathcal{B}$ to a brane $\mathcal{G}$ does not admit a lift to a string from the binding brane $\mathcal{C}$ to $\mathcal{G}$. Dually, not all the strings stretching from $\mathcal{F}$ to $\mathcal{D}$ can be extended to strings to $\mathcal{C}$. The obstructions for these lifts and extensions are described in Proposition 2.1.

In Proposition 2.4, we relate the vertex operators for strings ending on the brane $\mathcal{C}$ and the cohomology of other objects of $D(X)$ determined by the triangle. Under additional hypotheses, the result stated in Proposition 2.4 adopts the simpler form which appears in Proposition 2.7.

Section 3 concerns branes defined by coherent sheaves. As the category $\mathfrak{C o h}(X)$ is abelian, it is possible to define in $\mathfrak{C o h}(X)$ the homological concept of extension of an object by other [15]. This fact allows us to study the groups of strings between two coherent sheaves $\mathcal{F}$ and $\mathcal{G}$ in terms of extensions, without resorting to injective resolutions of $\mathcal{G}$. By interpreting the strings as extensions:

(i) We can show easily the definition of the bifunctors $E x t^{k}(.,$.$) on morphisms between coherent$ sheaves. The corresponding group homomorphisms give relations among different string spaces.

(ii) We can describe some strings of ghost number $k+1$ as obstructions on strings with $k$ ghost number.

To illustrate item (i), let us consider locally free sheaves $\mathcal{F}_{i}, i=1,2,3$, with $\mathcal{F}_{i}$ the sheaf of homolorphic sections of the vector bundle $V_{i} \rightarrow X$. Then, by Proposition 2.6, $\operatorname{Ext}^{k}\left(\mathcal{F}_{1}, \mathcal{F}_{2}\right)$ is the $k$ th cohomology group of $X$ with coefficients in the vector bundle $\operatorname{Hom}\left(V_{1}, V_{2}\right)$. So, given a gauge transformation $V_{2} \rightarrow V_{3}$, it induces an obvious homomorphism $\operatorname{Ext}^{k}\left(\mathcal{F}_{1}, \mathcal{F}_{2}\right) \rightarrow \operatorname{Ext}\left(\mathcal{F}_{1}, \mathcal{F}_{3}\right)$. However, when the $\mathcal{F}_{i}$ are general coherent sheaves the homomorphism between the corresponding Ext groups is not so evident. Nevertheless, in terms of extensions, the passage from $\operatorname{Ext}^{k}\left(\mathcal{F}_{1}, \mathcal{F}_{2}\right)$ to $\operatorname{Ext}^{k}\left(\mathcal{F}_{1}, \mathcal{F}_{3}\right)$ reduces to the construction of a fibred coproduct.

With respect to item (ii), given the subsheaf $\mathcal{B} \subset \mathcal{C}$, an object $\mathcal{G}$ of $\mathfrak{C o h}(X)$ and a string $\mathbf{R}$ with ghost number $p$ between $\mathcal{B}$ and $\mathcal{G}$, we construct an string of $\operatorname{Ext}^{p+1}(\mathcal{C} / \mathcal{B}, \mathcal{G})$ which is the obstruction for an extension of $\mathbf{R}$ to an string stretching between $\mathcal{C}$ and $\mathcal{G}$. Dually, some strings in $\operatorname{Ext}^{p+1}(\mathcal{F}, \mathcal{B})$, when $\mathcal{F}$ is a coherent sheaf, can be regarded as obstructions for the lift of elements in $\operatorname{Ext}^{p}(\mathcal{F}, \mathcal{C} / \mathcal{B})$ to strings from $\mathcal{F}$ to $\mathcal{C}$ (see Proposition 3.3).

If $X$ is an algebraic variety and it has a positive line bundle, then each coherent sheaf admits a global syzygy. This fact will permit us to describe the spaces of vertex operators as local extensions. More precisely, given the coherent sheaves $\mathcal{F}$ and $\mathcal{G}$, the vertex operators for strings between the branes $\mathcal{F}$ and $\mathcal{G}$ are the local extensions of the sheaf $\mathcal{G}$ by $\mathcal{F}$ (see Theorems 3.4 and 3.5). However, when $\mathcal{F}$ and $\mathcal{G}$ are $D 0$ branes the local extensions of $\mathcal{G}$ by $\mathcal{F}$ are, in fact, global; in other words, the spaces of local operators are isomorphic with the corresponding spaces of strings (see Example after Theorem 3.5).

To summarize, we enumerate some novel points we deal in this paper, which have not been considered hitherto in the literature. 
1. The introduction of the spaces of vertex operators for strings with ghost number different from 0 .

2. The application of cohomological methods for the interpretation of spaces of strings as obstructions to extensions or liftings.

3. The definition of the correlation functions for cohomological vertex operators.

4. The interpretation of the vertex operators as local extensions.

\section{Vertex Operators}

We will denote by $\mathcal{O}$ the sheaf of germs of holomorphic functions on $X$, and we will put $\mathcal{A}^{p, q}$ for the sheaf of germs of differential forms on $X$ of type $(p, q)$. By the Poincaré lemma relative to the operator $\bar{\partial}$, one has the following well-known resolution of the sheaf $\mathcal{O}$

$$
0 \rightarrow \mathcal{O} \rightarrow \mathcal{A}^{0,0} \stackrel{\bar{\partial}}{\rightarrow} \mathcal{A}^{0,1} \stackrel{\bar{\partial}}{\rightarrow} \mathcal{A}^{0,2} \rightarrow \ldots
$$

Let $V_{1}$ and $V_{2}$ be holomorphic vector bundles on $X$. We denote by $V$ the holomorphic vector bundle $\operatorname{Hom}\left(V_{1}, V_{2}\right)$, and by $\mathcal{O}(V)$ the sheaf of germs of holomorphic sections of $V$. We have also the corresponding sheaves of $V$-valued elements

$$
\mathcal{A}^{0, q}(V)=\mathcal{O}(V) \otimes \mathcal{O} \mathcal{A}^{0, q} .
$$

As the sheaf $\mathcal{O}(V)$ is locally free, the tensor product of $\mathcal{O}(V)$ by the resolution (3) gives the resolution

$$
0 \rightarrow \mathcal{O}(V) \longrightarrow \mathcal{A}^{0,0}(V) \stackrel{1 \otimes \bar{\partial}}{\longrightarrow} \mathcal{A}^{0,1}(V) \stackrel{1 \otimes \bar{Q}}{\longrightarrow} \mathcal{A}^{0,2}(V) \longrightarrow \ldots
$$

We put $A^{p, q}(V):=\Gamma\left(X, \mathcal{A}^{p, q}(V)\right)$ for the space of sections of the corresponding sheaf. As (4) is a fine resolution of $\mathcal{O}(V)$, one has

$$
H^{q}(X, \mathcal{O}(V))=h^{q}\left(A^{0, \bullet}(V)\right)
$$

That is,

$$
H^{q}\left(X, \mathcal{O}\left(\operatorname{Hom}\left(V_{1}, V_{2}\right)\right)\right)=h^{q}\left(A^{0, \bullet}\left(\operatorname{Hom}\left(V_{1}, V_{2}\right)\right)\right),
$$

where the right hand side is the $q$ th cohomology object of the complex $\left.A^{0, \bullet} \operatorname{Hom}\left(V_{1}, V_{2}\right)\right)$; in other words, the space of vertex operators $(1)$.

Thus, the elements of $H^{q}\left(X, \mathcal{O}\left(\operatorname{Hom}\left(V_{1}, V_{2}\right)\right)\right)$ can be considered as local operators, and the space of vertex operators for a string between $V_{1}$ and $V_{2}$ is the following direct sum of cohomology groups [2, page $207]$

$$
\bigoplus_{q} H^{q}\left(X, \mathcal{O}\left(\operatorname{Hom}\left(V_{1}, V_{2}\right)\right)\right)
$$

We will write the space (7) in other equivalent form, which admits a natural generalization to branes which are not locally free sheaves. For this purpose, we recall some properties of the functor Ext. We set Hom $(.,$.$) for the sheaf functor Hom (see [13, page 87])$

$$
\mathcal{H o m}(., .): \mathfrak{C o h}(X)^{\text {op }} \times \mathfrak{C o h}(X) \rightarrow \mathfrak{S h}
$$

where $\mathfrak{S h}$ is the category of sheaves of $\mathbb{C}$-vector spaces on $X$. It is easy to check that

$$
\mathcal{H o m}\left(\mathcal{O}\left(V_{1}\right), \mathcal{O}\left(V_{2}\right)\right)=\mathcal{O}\left(\operatorname{Hom}\left(V_{1}, V_{2}\right)\right)
$$

As we said, the bounded derived category of $\mathfrak{C o h}(X)$ will be denoted by $D(X)$, thus one has the derived functor

$$
R \mathcal{H o m}(., .): D(X)^{\mathrm{op}} \times D(X) \rightarrow D(\mathfrak{S h}),
$$

where $D(\mathfrak{S h})$ is the derived category of $\mathfrak{S h}$. By definition $\left.\mathcal{E} x t^{k}(\mathcal{F}, \mathcal{G})=H^{k} R \mathcal{H} o m(\mathcal{F}, \mathcal{G})\right)$.

On the other hand, we set Hom for the corresponding Hom functor of the category $\mathfrak{C o h}(X)$; so, denoting with $\mathfrak{V e c}$ the category of $\mathbb{C}$-vector spaces,

$$
\operatorname{Hom}(., .): \mathfrak{C o h}(X)^{\mathrm{op}} \times \mathfrak{C o h}(X) \rightarrow \mathfrak{V} \mathfrak{e c}
$$


Its derived functor

$$
R \operatorname{Hom}(., .): D(X)^{\text {op }} \times D(X) \rightarrow D(\mathfrak{V} \mathfrak{e c}),
$$

determines the Ext groups [7, page 194]

$$
\operatorname{Ext}^{k}(\mathcal{F}, \mathcal{G})=H^{k} R \operatorname{Hom}(\mathcal{F}, \mathcal{G}) .
$$

This is the space of strings with ghost number $k$ between the branes $\mathcal{F}$ and $\mathcal{G}$. Since

$$
H^{k} \operatorname{RHom}(\mathcal{F}, \mathcal{G})=\operatorname{Hom}_{D(X)}(\mathcal{F}, \mathcal{G}[k]),
$$

where $\mathcal{G}[k]$ is the complex $\mathcal{G}$ shifted by $k$ to the left, the strings between two branes can be considered as morphisms of the derived category $D(X)$.

Obviously, the $\mathcal{O}\left(V_{i}\right)$ are objects of $D(X)$. The equality

$$
\mathcal{H o m}\left(\mathcal{O}\left(V_{1}\right), \mathcal{O}\left(V_{2}\right)\right)=\mathcal{E} x t^{0}\left(\mathcal{O}\left(V_{1}\right), \mathcal{O}\left(V_{2}\right)\right)
$$

together with (8), allows us to write (7) as

$$
\bigoplus_{q} H^{q}\left(X, \mathcal{E} x t^{0}\left(\mathcal{O}\left(V_{1}\right), \mathcal{O}\left(V_{2}\right)\right)\right)
$$

To sum up, this is space of local operators for strings in

$$
\operatorname{Hom}\left(\mathcal{O}\left(V_{1}\right), \mathcal{O}\left(V_{2}\right)\right)=\operatorname{Ext}^{0}\left(\mathcal{O}\left(V_{1}\right), \mathcal{O}\left(V_{2}\right)\right)
$$

Therefore, the natural generalization of (7) for the space of vertex operators for strings with ghost number $k$ stretching between the branes $\mathcal{F}$ and $\mathcal{G}$ is

$$
\bigoplus_{q} H^{q}\left(X, \mathcal{E} x t^{k}(\mathcal{F}, \mathcal{G})\right)
$$

In particular, for a string with ghost number 0 stretching from $\mathcal{O}$ to $\mathcal{G}=\mathcal{O}\left(\bigwedge^{p} T X\right)$, one has the space of local operators

$$
H^{q}\left(X, \mathcal{E} x t^{0}(\mathcal{O}, \mathcal{G})\right)
$$

As $\mathcal{H o m}(\mathcal{O},$.$) is the identity functor, this space coincides with BRST cohomology group of the sigma$ $B$-model mentioned in Section 1 .

A consequence of the Grothendieck spectral sequence [19, page 403] [7, page 207] is the known Local-to-Global Ext spectral sequence, which allows to determine the Ext groups from the sheaves $\mathcal{E} x t$. That is, given the objects $\mathcal{F}$ and $\mathcal{G}$ in $D(X)$, the spectral sequence

$$
E_{2}^{p, q}=H^{p}\left(X, \mathcal{E} x t^{q}(\mathcal{F}, \mathcal{G})\right)
$$

abuts to $\operatorname{Ext} t^{k}(\mathcal{F}, \mathcal{G})$. Thus, the spaces of strings $\operatorname{Ext}^{k}(\mathcal{F}, \mathcal{G})$ are the limit of the spectral sequence determined by the space of vertex operators for strings between $\mathcal{F}$ and $\mathcal{G}$.

\subsection{Correlation Functions}

If $X$ is a projective variety of dimension $n$, the Serre functor $\mathcal{S}[4]$ in the category $D(X)$ is

$$
\mathcal{S}=(.) \otimes \omega_{X}[n]: D(X) \rightarrow D(X)
$$

where $\omega_{X}$ is the canonical sheaf of $X$ and $[n]$ denotes the shifting of the complex by $n$ to the left. Thus, for any $\mathcal{F}, \mathcal{H}$ objects of $D(X)$, one has a perfect pairing

$$
\operatorname{Hom}_{D(X)}(\mathcal{F}, \mathcal{H}) \otimes \operatorname{Hom}_{D(X)}\left(\mathcal{H}, \mathcal{F} \otimes \omega_{X}[n]\right) \rightarrow \mathbb{C} .
$$


Since $\mathcal{F} \otimes \omega_{X}[n]=\left(\mathcal{F} \otimes \omega_{X}\right)[n]$, the above pairing when $\mathcal{H}=\mathcal{F}[n]$ gives

$$
F: \operatorname{Ext}^{n}(\mathcal{F}, \mathcal{F}) \otimes \operatorname{Hom}_{D(X)}\left(\mathcal{F}, \mathcal{F} \otimes \omega_{X}\right) \rightarrow \mathbb{C} .
$$

When $X$ is a Calabi-Yau variety, as the canonical bundle of $X$ is trivial, there is an $n$-holomorphic form $\Omega$ which vanishes nowhere. $\Omega$ is unique up to multiplicative constant, and it can be fixed imposing

$$
\int_{X} \Omega \wedge \bar{\Omega}=1 .
$$

On the other hand, given an open subset $U$ of $X$, we put

$$
\epsilon(U): s \in \mathcal{F}(U) \mapsto s \otimes \Omega_{\mid U} \in \mathcal{F}(U) \otimes_{\mathcal{O}(U)} \omega_{X}(U)
$$

Then the maps $\epsilon(U)$ determine a morphism $\epsilon: \mathcal{F} \rightarrow \mathcal{F} \otimes \omega_{X}$, which in turn defines an element $\epsilon$ of $\operatorname{Hom}_{D(X)}\left(\mathcal{F}, \mathcal{F} \otimes \omega_{X}\right)$. So, we have the map

$$
t: \operatorname{Ext}^{n}(\mathcal{F}, \mathcal{F}) \rightarrow \mathbb{C}
$$

where $t(\sigma)$ is $F(\sigma \otimes \epsilon)$.

The element $E_{\infty}^{q, p}$ corresponding to the spectral sequence (14) is a subquotient of $E_{2}^{q, p}$. That is, there exists a tower of subspaces

$$
0=B_{1}^{p, q} \subset B_{2}^{p, q} \subset \cdots \subset Z_{2}^{p, q} \subset Z_{1}^{p, q}=E_{2}^{p, q},
$$

and

$$
E_{\infty}^{p, q}=Z_{\infty}^{p, q} / B_{\infty}^{p, q} ; \quad Z_{\infty}^{p, q}=\cap_{r} Z_{r}^{p, q}, \quad B_{\infty}^{p, q}=\cup_{r} B_{r}^{p, q} .
$$

Given the local operator $a \in H^{q}\left(X, \mathcal{E} x t^{p}(\mathcal{F}, \mathcal{G})\right)$, we define

$$
\alpha=\left\{\begin{array}{l}
{[a] \in E_{\infty}^{p, q}, \quad \text { if } a \in Z_{\infty}^{p, q}} \\
0 \in E_{\infty}^{p, q}, \quad \text { otherwise. }
\end{array}\right.
$$

As the sequence $(14)$ converges to $\operatorname{Ext} t^{q+p}(\mathcal{F}, \mathcal{G})$, the element $\alpha$ is a string with ghost number $p+q$ stretching between $\mathcal{F}$ and $\mathcal{G} ; \alpha \in \operatorname{Ext}^{q+p}(\mathcal{F}, \mathcal{G})$.

In particular, given the local operators $a_{j} \in H^{q_{j}}\left(X, \mathcal{E} x t^{p_{j}}\left(\mathcal{F}_{j-1}, \mathcal{F}_{j}\right)\right)$ for $j=1, \ldots, k$, satisfying $\sum_{j}\left(q_{j}+p_{j}\right)=n$ and $\mathcal{F}_{k}=\mathcal{F}_{0}$, then the Yoneda composite of the respective $\alpha_{j}$

$$
\alpha_{1} \star \cdots \star \alpha_{k} \in \operatorname{Ext}^{n}\left(\mathcal{F}_{0}, \mathcal{F}_{0}\right) .
$$

The $k$-correlation function for the local operators $a_{1}, \ldots, a_{k}$ can be defined as the complex number

$$
\left\langle a_{1} \ldots a_{k}\right\rangle:=t\left(\alpha_{1} \star \cdots \star \alpha_{k}\right) .
$$

\subsection{Distinguished Triangles and Obstructions}

Given the distinguished triangle $(2)$, as the functors $\operatorname{Hom}_{D(X)}(\mathcal{F},$.$) and \operatorname{Hom}_{D(X)}(., \mathcal{G})$ are cohomological functors, the triangle (2) gives rise to the long exact sequences of groups

$$
\begin{aligned}
& \cdots \rightarrow \operatorname{Ext}^{p}(\mathcal{F}, \mathcal{B}) \rightarrow \operatorname{Ext}^{p}(\mathcal{F}, \mathcal{C}) \rightarrow \operatorname{Ext}^{p}(\mathcal{F}, \mathcal{D}) \rightarrow \operatorname{Ext}^{p+1}(\mathcal{F}, \mathcal{B}) \rightarrow \ldots \\
& \cdots \rightarrow \operatorname{Ext}^{p}(\mathcal{D}, \mathcal{G}) \rightarrow \operatorname{Ext}^{p}(\mathcal{C}, \mathcal{G}) \rightarrow \operatorname{Ext}^{p}(\mathcal{B}, \mathcal{G}) \rightarrow \operatorname{Ext}^{p+1}(\mathcal{D}, \mathcal{G}) \rightarrow \ldots
\end{aligned}
$$

where $\mathcal{F}$ and $\mathcal{G}$ are objects of $D(X)$. Thus, we have the following proposition.

Proposition 2.1. Let us assume that the brane $\mathcal{C}$ can decay into the branes $\mathcal{B}$ and $\mathcal{D}$, according to (2).

1. If $\tau$ is a string between $\mathcal{F}$ and $\mathcal{D}$ with ghost number $p$, then $\operatorname{Ext}^{p+1}(\mathcal{F}, \tau)$ is the obstruction for a lift of $\tau$ to a string stretching from $\mathcal{F}$ to $\mathcal{C}$. 
2. If $\rho$ is a string in $\operatorname{Ext}^{p}(\mathcal{B}, \mathcal{G})$, then $\operatorname{Ext}^{p+1}(\rho, \mathcal{G})$ is the obstruction for an extension of $\rho$ to a string between $\mathcal{C}$ and $\mathcal{G}$.

Roughly speaking, $\operatorname{Ext}^{p+1}(\mathcal{D}, \mathcal{G})$ computes the strings from $\mathcal{B}$ to $\mathcal{G}$, with ghost number $p$, which can not be "extended" to strings between $\mathcal{C}$ and $\mathcal{G}$. Dually, $\operatorname{Ext}^{p+1}(\mathcal{F}, \mathcal{B})$ computes the strings stretching from $\mathcal{F}$ to $\mathcal{D}$, with ghost number $p$, which can not be "lifted" to strings between $\mathcal{F}$ and $\mathcal{C}$.

Given the distinguished triangle $(2)$ in $D(X)$ and an object $\mathcal{F}$ of this category, we will show that the spaces of vertex operators $\left\{H^{q}\left(X, \mathcal{E} x t^{k}(\mathcal{F}, \mathcal{C})\right)\right\}_{q}$ (with $k$ fixed) can be included in a long exact sequence with other spaces of local operators determined by the vertices of the triangle.

To deduce that exact sequence, we will prove that the functor $\operatorname{RHom}(\mathcal{F},):. D(X) \rightarrow D(\mathfrak{S h})$ is $t$-exact, i.e., it transforms distinguished triangles into distinguished triangles [7, page 285].

Returning to the triangle $(2)$, if $\mathcal{P}$ is a complex in the category of $\mathcal{O}$-modules quasi-isomorphic to $\mathcal{F}$ with $\mathcal{P}^{i}$ a projective object, then

$$
\mathcal{E} x t^{k}(\mathcal{F}, \mathcal{B})=h^{k}(\mathcal{H o m} \bullet(\mathcal{P}, \mathcal{B}))
$$

where $\mathcal{H o m}^{\bullet}(\mathcal{P}, \mathcal{B})$ is the complex of $\mathcal{O}$-modules, defined by

$$
\mathcal{H o m}^{n}(\mathcal{P}, \mathcal{B})=\prod_{i} \mathcal{H o m}\left(\mathcal{P}^{i}, \mathcal{B}^{i+n}\right)
$$

(Dually, we could take an injective resolution of $\mathcal{B}$, such injective resolutions always exist in the category of $\mathcal{O}$-modules).

Let $\mathfrak{C}$ denote the category of complexes of $\mathcal{O}$-modules. With respect to the triangle $(2)$, it is not restrictive to assume that $\mathcal{C}$ and $\mathcal{D}$ are the mappings cylinder and cone of some morphism $g$ of $\mathfrak{C}$ with domain $\mathcal{B}$; that is,

$$
\mathcal{C}=\operatorname{Cyl}(g), \mathcal{D}=\operatorname{Con}(g),
$$

and the morphisms $u$ and $v$ are the natural ones (see Proposition 8 in page 256 of [7]).

In the Appendix, we will prove the following proposition.

Proposition 2.2. Let $U$ be an open subset of $X$, then

$$
\mathcal{H o m}^{\bullet}(\mathcal{P}, \operatorname{Con}(g))(U)=\operatorname{Con}(\widehat{g})(U), \mathcal{H o m}^{\bullet}(\mathcal{P}, \operatorname{Cyl}(g))(U)=\operatorname{Cyl}(\widehat{g})(U),
$$

$\widehat{g}$ being $\mathcal{H o m}^{\bullet}(\mathcal{P}, g)$.

Applying the functor $\mathcal{H o m}^{\bullet}(\mathcal{P},$.$) to the triangle (2) and taking into account (20), one obtains the$ sequence

$$
\begin{aligned}
& \operatorname{Hom}^{\bullet}(\mathcal{P}, \mathcal{B}) \rightarrow \mathcal{H o m}^{\bullet}(\mathcal{P}, \operatorname{Cyl}(g)) \rightarrow \mathcal{H o m}^{\bullet}(\mathcal{P}, \operatorname{Con}(g)) \rightarrow \\
& \rightarrow \mathcal{H o m}^{\bullet}(\mathcal{P}, \mathcal{B}[1]) .
\end{aligned}
$$

By Proposition 2.2, for any open $U$, we have

$$
\begin{aligned}
& \mathcal{H o m}^{\bullet}(\mathcal{P}, \mathcal{B})(U) \rightarrow \operatorname{Cyl}(\widehat{g})(U) \rightarrow \operatorname{Con}(\widehat{g})(U) \rightarrow \\
& \mathcal{H o m}^{\bullet}(\mathcal{P}, \mathcal{B}[1])(U)=\mathcal{H o m}^{\bullet}(\mathcal{P}, \mathcal{B})[1](U) .
\end{aligned}
$$

Hence, one has the following distinguished triangle in the category $D(X)$

$$
\mathcal{H o m}^{\bullet}(\mathcal{P}, \mathcal{B}) \rightarrow \operatorname{Cyl}(\widehat{g}) \rightarrow \operatorname{Con}(\widehat{g}) \rightarrow \mathcal{H o m}^{\bullet}(\mathcal{P}, \mathcal{B})[1]
$$

We have proved the following proposition.

Proposition 2.3. The functor $\operatorname{RHom}(\mathcal{F},$.$) is t-exact.$ 
Similarly, the functor $R \mathcal{H}$ om $(., \mathcal{F})$ are $t$-exact.

As the functor $H^{0}$ is cohomological in any triangulated category [7, page 283], from the distinguished triangle (2) in $\mathrm{D}(\mathrm{X})$, by Proposition 2.3 , one obtains the exact sequence

$$
\cdots \rightarrow \mathcal{E} x t^{k}(\mathcal{F}, \mathcal{B}) \stackrel{\alpha}{\rightarrow} \mathcal{E} x t^{k}(\mathcal{F}, \mathcal{C}) \stackrel{\beta}{\rightarrow} \mathcal{E} x t^{k}(\mathcal{F}, \mathcal{D}) \stackrel{\gamma}{\rightarrow} \mathcal{E} x t^{k+1}(\mathcal{F}, \mathcal{B}) \rightarrow \ldots
$$

in the category $\mathfrak{S h}$ of sheaves of $\mathbb{C}$-vector spaces on $X$.

The following proposition yields the mentioned exact sequence, in which the space of vertex operators $\left\{H^{q}\left(X, \mathcal{E} x t^{k}(\mathcal{F}, \mathcal{C})\right)\right\}_{q}$ are involved.

Proposition 2.4. Given the distinguished triangle (2), the following cohomology sequence is exact

$$
\begin{aligned}
\ldots & \rightarrow H^{q}(X, \operatorname{Ker}(\beta)) \rightarrow H^{q}\left(X, \mathcal{E} x t^{k}(\mathcal{F}, \mathcal{C})\right) \rightarrow H^{q}(X, \operatorname{Im}(\beta)) \rightarrow \\
& \rightarrow H^{q+1}(X, \operatorname{Ker}(\beta)) \rightarrow \ldots
\end{aligned}
$$

where $\beta: \mathcal{E} x t^{k}(\mathcal{F}, \mathcal{C}) \rightarrow \mathcal{E} x t^{k}(\mathcal{F}, \mathcal{D})$ is the morphism induced by the arrow $v$ of (2).

Proof. From (21), one deduces the short exact sequence of sheaves

$$
0 \rightarrow \operatorname{Ker}(\beta) \rightarrow \mathcal{E} x t^{k}(\mathcal{F}, \mathcal{C}) \rightarrow \operatorname{Im}(\beta) \rightarrow 0 .
$$

The sequence (22) is the corresponding long exact cohomology sequence.

\subsection{Vertex Operators for Strings between Locally Free Sheaves}

If $\mathcal{E}$ is a locally free $\mathcal{O}$-module, then one has the following locally free resolution $\mathcal{E}_{\bullet}$ of $\mathcal{E}$, where $\mathcal{E}_{i}=0$ for $i \neq 0$ and $\mathcal{E}_{0}=\mathcal{E}$

$$
\cdots \rightarrow 0 \rightarrow 0 \rightarrow \mathcal{E} \rightarrow \mathcal{E} \rightarrow 0
$$

which can be used to construct the sheaves $\mathcal{E} x t^{k}(\mathcal{E}, \mathcal{G})$, with $\mathcal{G}$ any coherent $\mathcal{O}$-module (see Proposition 6.5 in page 234 of [9]). Hence,

$$
\mathcal{E} x t^{p}(\mathcal{E}, \mathcal{G})=h^{p}\left(\mathcal{H o m}\left(\mathcal{E}_{\bullet}, \mathcal{G}\right)\right)=0, \text { for } p>0
$$

So, we have the proposition.

Proposition 2.5. Let $\mathcal{E}$ be a locally free $\mathcal{O}$-module and $\mathcal{G}$ an arbitrary coherent $\mathcal{O}$-module. Then, the space (13) of vertex operators for a string of $\operatorname{Ext}^{k}(\mathcal{E}, \mathcal{G})$ is zero, when $k>0$.

Thus, by the proposition, to get nontrivial local operators for strings with ghost number $k>0$, it is necessary to consider more general branes, for instance coherent not locally free sheaves on $X$. Among those are the quotient $\mathcal{O} / \mathcal{I}$ of $\mathcal{O}$ by an ideal sheaf.

Under the hypotheses of the proposition, the Local-to-Global spectral sequence is also trivial. Then, one has the following result.

Proposition 2.6. Under the hypotheses of Proposition 2.5,

$$
\operatorname{Ext}^{k}(\mathcal{E}, \mathcal{G})=H^{k}(X, \mathcal{H o m}(\mathcal{E}, \mathcal{G}))
$$

That is, the strings with ghost number $k$ starting from a locally free $\mathcal{O}$-module are vertex operators for strings with ghost number 0 .

Distinguished triangles. Next, we will consider Proposition 2.4, when in the triangle (2) $\mathcal{B}, \mathcal{C}$ and $\mathcal{D}$ are $\mathcal{O}$-modules. If $\mathcal{E}$ is a locally free sheaf, by Proposition 2.6, the exact sequences (17) and (18) can be written in terms of vertex operators. Thus, one obtains the following proposition which relates the spaces of vertex operators for strings which end at the branes $\mathcal{B}, \mathcal{C}, \mathcal{D}$ or begin in these branes. 
Proposition 2.7. Let $\mathcal{B} \rightarrow \mathcal{C} \rightarrow \mathcal{D} \stackrel{+1}{\rightarrow} \mathcal{B}[1]$ be a distinguished triangle consisting of $\mathcal{O}$-modules, and $\mathcal{E}$ a locally free $\mathcal{O}$-module. Then the following sequences of vertex operators are exact

$$
\begin{aligned}
\ldots & \rightarrow H^{q}(X, \mathcal{H o m}(\mathcal{E}, \mathcal{B})) \rightarrow H^{q}(X, \mathcal{H o m}(\mathcal{E}, \mathcal{C})) \rightarrow \\
& \rightarrow H^{q}(X, \mathcal{H o m}(\mathcal{E}, \mathcal{D})) \rightarrow H^{q+1}(X, \mathcal{H o m}(\mathcal{E}, \mathcal{B})) \rightarrow \ldots
\end{aligned}
$$

and

$$
\begin{aligned}
\ldots & \rightarrow H^{q}(X, \mathcal{H o m}(\mathcal{D}, \mathcal{E})) \rightarrow H^{q}(X, \mathcal{H o m}(\mathcal{C}, \mathcal{E})) \rightarrow \\
& \rightarrow H^{q}(X, \mathcal{H o m}(\mathcal{B}, \mathcal{E})) \rightarrow H^{q+1}(X, \mathcal{H o m}(\mathcal{D}, \mathcal{E})) \rightarrow \ldots
\end{aligned}
$$

Correlation functions. Next, we will deduce the form adopted by the correlation function (16) for strings between locally free $\mathcal{O}$-modules. For this deduction, the following lemma will be useful.

Lemma 2.8. Let $V$ be a holomorphic vector bundle on $X$ and $\mathcal{F}=\mathcal{O}(V)$, then the map $t$ defined in (15) reduces to

$$
t(\sigma)=\int_{X} \operatorname{tr}(\sigma) \wedge \Omega
$$

$\sigma \in H_{\bar{\partial}}^{0, n}(X, \operatorname{End}(V))$.

Proof. If $V$ is a holomorphic vector bundle on $X$, then the Serre duality gives the pairing

$$
H^{q}(X, V) \otimes H^{n-q}\left(X, \omega_{X} \otimes V^{*}\right) \rightarrow \mathbb{C}, \quad \alpha \otimes \beta \mapsto \int_{X} \alpha \wedge \beta,
$$

where in $\alpha \wedge \beta$ the tautological paring on $V_{x} \otimes V_{x}^{*}$ is involved; i.e., the trace on $\operatorname{End}\left(V_{x}\right)$. Thus, by Proposition 2.6 together with (6) the map $t$ in this case takes the form

$$
\sigma \in H_{\bar{\partial}}^{0, n}(X, \operatorname{End}(V))=\operatorname{Ext}^{n}(\mathcal{O}(V), \mathcal{O}(V)) \mapsto \int_{X} \operatorname{tr}(\sigma) \wedge \Omega .
$$

Let $V_{i}, i=1, \ldots, k$ be holomorphic vector bundles on $X$, we put $\mathcal{F}_{j}=\mathcal{O}\left(V_{j}\right)$. By Proposition 2.6 together with (6)

$$
H_{\bar{\partial}}^{0, q}\left(X, \operatorname{Hom}\left(V_{i}, V_{j}\right)\right)=\operatorname{Ext}^{q}\left(\mathcal{F}_{i}, \mathcal{F}_{j}\right)
$$

The composition pairing between the holomorphic vector bundles

$$
\operatorname{Hom}\left(V_{i}, V_{j}\right) \otimes_{\mathcal{O}} \operatorname{Hom}\left(V_{j}, V_{r}\right) \rightarrow \operatorname{Hom}\left(V_{i}, V_{r}\right)
$$

gives rise to the Yoneda pairing [8, page 707], which, by (24), adopts the following form

$$
\begin{gathered}
H_{\bar{\partial}}^{0, p}\left(X, \operatorname{Hom}\left(V_{i}, V_{j}\right)\right) \otimes_{\mathcal{O}} H_{\bar{\partial}}^{0, q}\left(X, \operatorname{Hom}\left(V_{j}, V_{r}\right)\right) \stackrel{\star}{\longrightarrow} \\
H_{\bar{\partial}}^{0, p+q}\left(X, \operatorname{Hom}\left(V_{i}, V_{r}\right)\right) .
\end{gathered}
$$

The cup product and the composition of homomorphisms are involved in the definition of $\star$.

By Proposition 2.5, to have nonzero local operators

$$
a_{j} \in H^{q_{j}}\left(X, \mathcal{E}_{x} t^{p_{j}}\left(\mathcal{F}_{j-1}, \mathcal{F}_{j}\right)\right)
$$

it is necessary that $p_{j}=0$. And according to Proposition 2.6, the $\alpha_{j}$ 's in (16) are identical to the $a_{j}$ 's. Thus, given $\alpha_{i} \in \operatorname{Ext}^{q_{i}}\left(\mathcal{F}_{i-1}, \mathcal{F}_{i}\right)$, if $V_{0}=V_{k}$ and $\sum q_{i}=n$, then

$$
\alpha_{1} \star \cdots \star \alpha_{k} \in \operatorname{Ext}^{n}\left(\mathcal{F}_{0}, \mathcal{F}_{0}\right)=H_{\bar{\partial}}^{0, n}\left(X, \operatorname{Hom}\left(V_{0}, V_{0}\right)\right) \text {. }
$$


Proposition 2.9. Given the locally free sheaves $\left\{\mathcal{F}_{i}=\mathcal{O}\left(V_{i}\right)\right\}_{i=1, \ldots, k}$, with $V_{0}=V_{k}$ and the vertex operators $a_{j} \in H^{q_{j}}\left(X, \mathcal{E} x t^{0}\left(\mathcal{F}_{j-1}, \mathcal{F}_{j}\right)\right)$, such that $\sum q_{j}=n$. Then the correlation function

$$
\left\langle a_{1} \ldots a_{k}\right\rangle=\int_{X} \operatorname{tr}\left(\alpha_{1} \wedge \cdots \wedge \alpha_{k}\right) \wedge \Omega
$$

Proof. It is a direct consequence of the definition (16) and Lemma 2.8.

Therefore, the correlation function defined in (16) generalizes the one given in [2, page 208] for vertex operators which are elements in spaces of the form (6).

Remarks. We summarize the most important points considered in this section. We have introduced the vertex operators on the manifold $X$ as elements of the cohomology groups of objects in the derived category of sheaves. For the case when $X$ is a projective variety, we have defined the correlation functions for the new operators, and we have proved that they generalize the usual ones for local operators associated to strings between locally free sheaves.

When the brane $\mathcal{C}$ may decay into the branes $\mathcal{B}$ and $\mathcal{D}$, we have proved that strings in $\operatorname{Ext}^{p+1}(\mathcal{F}, \mathcal{B})$ are the obstructions for the lift of strings from $\mathcal{F}$ to $\mathcal{D}$, with ghost number $p$, to strings between $\mathcal{F}$ to $\mathcal{C}$. Dually, strings in $\operatorname{Ext}^{p+1}(\mathcal{D}, \mathcal{G})$ are the obstructions for the extension of strings from $\mathcal{B}$ to $\mathcal{G}$, with ghost number $p$, to strings between $\mathcal{C}$ to $\mathcal{G}$.

We have also studied the relations between the following sets vertex operators

$$
\left\{H^{q}(X, \mathcal{H o m}(\mathcal{E}, \mathcal{B}))\right\}_{q},\left\{H^{q}(X, \mathcal{H o m}(\mathcal{E}, \mathcal{C}))\right\}_{q},\left\{H^{q}(X, \mathcal{H o m}(\mathcal{E}, \mathcal{D}))\right\}_{q}
$$

when $\mathcal{E}$ is a locally free module.

\section{Strings between Coherent Sheaves}

In this section, we only consider branes which are objects of the category $\mathfrak{C o h}(X)$, that is, coherent sheaves on $X$. The category $\mathfrak{C o h}(X)$ has enough injectives; hence, the restriction of the functors $E x t^{k}$, defined in (10), to $\mathfrak{C o h}(X)$ coincides with the classical derived functors of Hom [19, Corollary 10.7.5]. That is, the spaces of strings (with a given ghost number) between coherent sheaves can be determined by calculating the derived functors of Hom without passing to the derived category.

As $\mathfrak{C o h}(X)$ is an abelian category, the Ext groups can be defined and studied in terms of extensions [15]. We will adopt this point of view in this section. In $\mathfrak{C o h}(X)$ it is possible to construct exact sequences, fibred products and coproducts. These tools will permit us to relate string spaces between different branes and analyze the obstructions to "extensions" and "lifts" mentioned in the preceding section.

The functor (9) is not exact. That is, if $\mathcal{B}$ is a sub-brane of $\mathcal{C}$, there are strings with ghost number 0 between two branes $\mathcal{B}$ and $\mathcal{G}$, which do not admit an extension to a string from $\mathcal{C}$ to $\mathcal{G}$. Dually, there are strings with 0 ghost number from $\mathcal{F}$ to the quotient $\mathcal{C} / \mathcal{B}$ which do not admit a lift to a string starting from $\mathcal{F}$. The "obstructions" to these processes are described by elements of $\operatorname{Ext}^{1}(\mathcal{C} / \mathcal{B}, \mathcal{G})$ in the first case, and by elements of $\operatorname{Ext}^{1}(\mathcal{F}, \mathcal{B})$ in the dual case. One can say that the functor $\operatorname{Ext}^{1}$ is the track of the inexactitude of the functor Hom. Similarly, Ext ${ }^{2}$ is consequence of the inexactitude of $E x t^{1}$, etc.

More precisely, the $E x t^{i}$ functors as right derived functors of the functor Hom form a $\delta$-functor [9, page 205]. Thus, given the short exact sequence $U$ in the abelian category $\mathfrak{C} o \mathfrak{h}(X)$ of coherent $\mathcal{O}$-modules

$$
U: \quad 0 \rightarrow \mathcal{B} \rightarrow \mathcal{C} \rightarrow \mathcal{D}=\mathcal{C} / \mathcal{B} \rightarrow 0
$$

one has the long exact sequences (17) and (18).

\subsection{Extensions of Coherent Sheaves}

Here, as we have said, one will consider the spaces of strings between two branes as groups of extensions of coherent $\mathcal{O}$-modules. 
A length $p$ extension of the coherent $\mathcal{O}$-module $\mathcal{G}$ by the coherent sheaf $\mathcal{F}$ is an exact sequence of $\mathcal{O}$-modules $[14$, page 63$]$

$$
\mathbf{S}: \quad 0 \rightarrow \mathcal{G} \rightarrow \mathcal{H}_{p-1} \rightarrow \mathcal{H}_{p-2} \rightarrow \cdots \rightarrow \mathcal{H}_{0} \rightarrow \mathcal{F} \rightarrow 0
$$

Given $S$ and $S^{\prime}$ two extensions of length 1 of $\mathcal{G}$ by $\mathcal{F}$, we say they are equivalent if there exists a morphism between the exact sequences $S$ and $S^{\prime}$ as the one showed in the following diagram

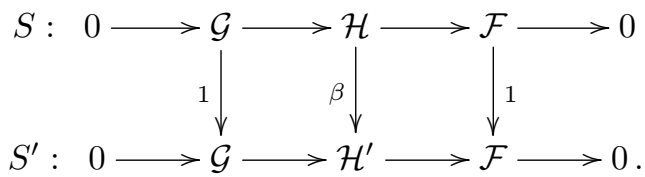

It is easy to check that $\beta$ must be an isomorphism; thus, we have defined an equivalence relation in the set of extensions of $\mathcal{G}$ by $\mathcal{F}$. The corresponding quotient space can be endowed with the structure of abelian group [14]. This group is denoted by $\operatorname{Ext}^{1}(\mathcal{F}, \mathcal{G})$ and is called the group of extensions of $\mathcal{G}$ by $\mathcal{F}$. The zero element of this group is defined by the extension

$$
0 \rightarrow \mathcal{G} \rightarrow \mathcal{G} \oplus \mathcal{F} \rightarrow \mathcal{F} \rightarrow 0
$$

Although $\operatorname{Ext}^{1}(\mathcal{F}, \mathcal{G})$ and $\operatorname{Ext} t^{1}(\mathcal{F}, \mathcal{G})$ are isomorphic, we will maintain the roman typos for the constructions of the Ext groups we will carry out in this subsection, and by notational consistency, we will put Hom for denoting Hom.

Given an 1 extension $S$ of $\mathcal{G}$ by $\mathcal{F}$ and a morphism $\gamma: \mathcal{F}^{\prime} \rightarrow \mathcal{F}$, the pullback construction permits to define an extension $S \gamma$ of $\mathcal{G}$ by $\mathcal{F}^{\prime}$ so that the following diagram is commutative

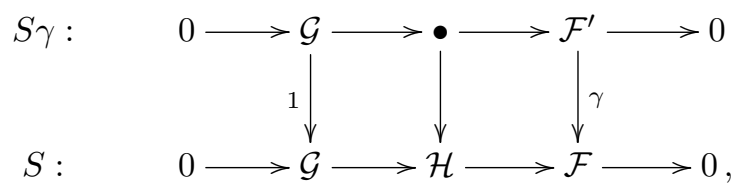

where the right hand square is the corresponding cartesian square. We have a group homomorphism

$$
S \in \operatorname{Ext}^{1}(\mathcal{F}, \mathcal{G}) \longrightarrow S \gamma \in \operatorname{Ext}^{1}\left(\mathcal{F}^{\prime}, \mathcal{G}\right)
$$

If $\mathcal{F}^{\prime}, \mathcal{F}$ and $\mathcal{H}$ are the sheaves of holomorphic sections of the vector bundles $F^{\prime}, F$ and $H$, respectively, then the sheaf represented by $\bullet$ in the diagram corresponds to the vector bundle whose fibre at the point $x \in X$ is

$$
\left\{\left(f^{\prime}, h\right) \in F_{x}^{\prime} \times H_{x} \mid \gamma_{x}\left(f^{\prime}\right)=\chi_{x}(h)\right\}
$$

where $\chi$ denotes the morphism $H \rightarrow F$.

Similarly, given $\alpha: \mathcal{G} \rightarrow \mathcal{G}^{\prime}$, one can define the extension $\alpha S$ of $\mathcal{G}^{\prime}$ by $\mathcal{F}$

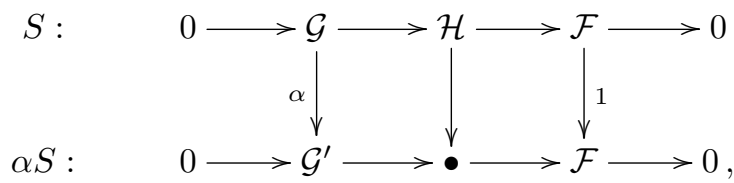

where the left hand square is the fibered coproduct. One has a group homomorphism

$$
S \in \operatorname{Ext}^{1}(\mathcal{F}, \mathcal{G}) \longrightarrow \alpha S \in \operatorname{Ext}^{1}\left(\mathcal{F}, \mathcal{G}^{\prime}\right) .
$$

The maps (28) and (29) show the functorial character of $\operatorname{Ext}^{1}(.,$.$) . The above operations are$ associative, in the sense that the extensions $(\alpha S) \gamma$ and $\alpha(S \gamma)$ are equivalent. Thus, if the branes are related by the morphisms $\alpha$ and $\gamma$ as above, we have the following relation between the corresponding spaces of strings

$$
S \in \operatorname{Ext}^{1}(\mathcal{F}, \mathcal{G}) \longrightarrow \alpha S \gamma \in \operatorname{Ext}^{1}\left(\mathcal{F}^{\prime}, \mathcal{G}^{\prime}\right)
$$


On the other hand, with fixed $S \in \operatorname{Ext}^{1}(\mathcal{F}, \mathcal{G})$, the preceding constructions can be regarded as maps

$$
\begin{aligned}
& \alpha \in \operatorname{Hom}\left(\mathcal{G}, \mathcal{G}^{\prime}\right) \rightarrow \alpha S \in \operatorname{Ext}^{1}\left(\mathcal{F}, \mathcal{G}^{\prime}\right) \\
& \gamma \in \operatorname{Hom}\left(\mathcal{F}^{\prime}, \mathcal{F}\right) \rightarrow S \gamma \in \operatorname{Ext}^{1}\left(\mathcal{F}^{\prime}, \mathcal{G}\right) .
\end{aligned}
$$

Given a sub-brane $\mathcal{B}$ of a brane of $\mathcal{C}$, one has the exact sequence $U$ defined in (26). The following proposition asserts that a morphism $\rho: \mathcal{B} \rightarrow \mathcal{G}$ can be extended to a morphisms defined on $\mathcal{C}$ if the short exact sequence $\rho U$ splits.

Proposition 3.1. Let $\mathcal{B}$ be a sub-brane of $\mathcal{C}$. The obstruction to an extension of the string $\rho \in \operatorname{Hom}(\mathcal{B}, \mathcal{G})$ to a string stretching from $\mathcal{C}$ to $\mathcal{G}$ is the string $\rho U \in \operatorname{Ext}^{1}(\mathcal{C} / \mathcal{B}, \mathcal{G})$.

Proof. From the long exact sequence of Ext goups, one obtains the following exact sequence

$$
\operatorname{Hom}(\mathcal{C}, \mathcal{G}) \rightarrow \operatorname{Hom}(\mathcal{B}, \mathcal{G}) \rightarrow \operatorname{Ext}^{1}(\mathcal{C} / \mathcal{B}, \mathcal{G})
$$

where the first arrow is the restriction homomorphism. The second map is, by (30), the correspondence $\rho \mapsto \rho U$.

Example. If $\mathcal{C}=\mathcal{O}$ and $\mathcal{B}$ is an ideal sheaf $\mathcal{I}$ of $\mathcal{O}$. Then $Z:=\operatorname{Supp}(\mathcal{O} / \mathcal{I})$ is an analytic subvariety of $X$ and we put $\mathcal{O}_{Z}$ for the coherent sheaf $\mathcal{O} / \mathcal{I}$.

Let $\mathcal{G}$ be a coherent $\mathcal{O}$-module, then $\mathcal{H o m}(\mathcal{O}, \mathcal{G})=\mathcal{G}$. By Proposition 2.6,

$$
\operatorname{Ext}^{1}(\mathcal{O}, \mathcal{G})=H^{1}(X, \mathcal{G})
$$

Obviously, the restriction of an element of $\operatorname{Hom}(\mathcal{O}, \mathcal{G})=\Gamma(X, \mathcal{G})$ determines a string from $\mathcal{I}$ to $\mathcal{G}$ with ghost number 0 .

If $H^{1}(X, \mathcal{G})=0$, then a part of the long exact sequence of Ext is

$$
\operatorname{Hom}(\mathcal{O}, \mathcal{G}) \rightarrow \operatorname{Hom}(\mathcal{I}, \mathcal{G}) \rightarrow \operatorname{Ext}^{1}\left(\mathcal{O}_{Z}, \mathcal{G}\right) \rightarrow 0
$$

Thus,

$$
\operatorname{Ext}^{1}\left(\mathcal{O}_{Z}, \mathcal{G}\right)=\operatorname{Hom}(\mathcal{I}, \mathcal{G}) / \operatorname{Hom}(\mathcal{O}, \mathcal{G})
$$

Roughly speaking, in $\operatorname{Ext}^{1}\left(\mathcal{O}_{Z}, \mathcal{G}\right)$ one computes the morphisms from $\mathcal{I}$ to $\mathcal{G}$ (i.e. strings stretching from $\mathcal{I}$ to $\mathcal{G}$ with 0 ghost number) that do not admit an extension to morphisms from $\mathcal{O}$ to $\mathcal{G}$, i. e. which are not defined by global sections of $\mathcal{G}$.

The result stated in Proposition 3.1 has the corresponding dual.

Proposition 3.2. Let $\mathcal{B}$ be a sub-brane of $\mathcal{C}$. The obstruction to a lift of the string $\tau \in \operatorname{Hom}(\mathcal{F}, \mathcal{C} / \mathcal{B})$ to a string stretching from $\mathcal{F}$ to $\mathcal{C}$ is the string $U \tau \in \operatorname{Ext}^{1}(\mathcal{F}, \mathcal{B})$.

Given the short exact sequences

$$
R: 0 \rightarrow \mathcal{G} \rightarrow \mathcal{H}_{1} \rightarrow \mathcal{K} \rightarrow 0, \quad R^{\prime}: 0 \rightarrow \mathcal{K} \rightarrow \mathcal{H}_{0} \rightarrow \mathcal{F} \rightarrow 0
$$

the following exact sequence is called the Yoneda composite of $R$ and $R^{\prime}$

$$
R \star R^{\prime}: 0 \rightarrow \mathcal{G} \rightarrow \mathcal{H}_{1} \rightarrow \mathcal{H}_{0} \rightarrow \mathcal{F} \rightarrow 0 .
$$

If $S$ and $S^{\prime}$ are short exact sequences and $\beta$ a morphism such that the the Yoneda composite $(S \beta) \star S^{\prime}$ is defined, then there is a morphism of exact sequences

$$
(S \beta) \star S^{\prime} \rightarrow S \star\left(\beta S^{\prime}\right)
$$

which in general is not an isomorphism.

The long exact sequence (27) can be written as a composition of short exact sequences

$$
\mathbf{S}=S_{p} \star S_{p-1} \star \cdots \star S_{1},
$$


by decomposing the maps of (27) in product of a monomorphism and an epimorphism.

One says that the sequences

$$
\mathbf{S}=S_{p} \star \cdots \star S_{1}, \quad \mathbf{S}^{\prime}=S_{p}^{\prime} \star \cdots \star S_{1}^{\prime},
$$

are equivalent if one can be obtained from the other through switches of the form (33). The quotient by this equivalence of the set of $p$ fold extensions of $\mathcal{G}$ by $\mathcal{F}$ can be endowed with structure of abelian group and is denoted $\operatorname{Ext}^{p}(\mathcal{F}, \mathcal{G})$. As it is known, the groups $\operatorname{Ext}^{p}(\mathcal{F}, \mathcal{G})$ are isomorphic to the values taken at $(\mathcal{F}, \mathcal{G})$ by the corresponding derived functors $\operatorname{Ext}^{p}$ defined in (10). So, the short exact sequence (26) gives rise to long exact sequences of Ext ${ }^{i}$ groups as in (17)-(18). Next, we will construct the morphisms of these exact sequences from operations with extensions.

Let $\alpha: \mathcal{G} \rightarrow \mathcal{G}^{\prime}$ and $\gamma: \mathcal{F}^{\prime} \rightarrow \mathcal{F}$ be the above morphisms of branes. If $\mathbf{S}$ is the extension (27), then one defines

$$
\alpha \mathbf{S}:=\left(\alpha S_{p}\right) \star \cdots \star S_{1} \in \operatorname{Ext}^{p}\left(\mathcal{F}, \mathcal{G}^{\prime}\right), \quad \mathbf{S} \gamma:=S_{p} \star \cdots \star\left(S_{1} \gamma\right) \in \operatorname{Ext}^{p}\left(\mathcal{F}^{\prime}, \mathcal{G}\right)
$$

Thus, the pair $(\gamma, \alpha)$ determines the following relation between the corresponding string spaces

$$
\mathbf{S} \in \operatorname{Ext}^{p}(\mathcal{F}, \mathcal{G}) \mapsto \alpha(\mathbf{S} \gamma) \in \operatorname{Ext}^{p}\left(\mathcal{F}^{\prime}, \mathcal{G}^{\prime}\right)
$$

On the other hand, given the following extension of $\mathcal{G}$ by $\mathcal{B}$

$$
\mathbf{R}: \quad 0 \rightarrow \mathcal{G} \rightarrow \mathcal{E}_{p-1} \rightarrow \mathcal{E}_{p-2} \rightarrow \cdots \rightarrow \mathcal{E}_{0} \rightarrow \mathcal{B} \rightarrow 0,
$$

composing (35) with (26) one obtaines the exact sequence

$$
\mathbf{R} \star U: \quad 0 \rightarrow \mathcal{G} \rightarrow \mathcal{E}_{p-1} \rightarrow \cdots \rightarrow \mathcal{E}_{0} \rightarrow \mathcal{C} \rightarrow \mathcal{D} \rightarrow 0,
$$

which determines an element of $\operatorname{Ext}^{p+1}(\mathcal{D}, \mathcal{G})$. The group homomorphism induced by the map $\mathbf{R} \mapsto \mathbf{R} \star U$ is the connecting homomorphism in the long exact sequence associated to (26)

$$
\cdots \rightarrow \operatorname{Ext}^{p}(\mathcal{D}, \mathcal{G}) \rightarrow \operatorname{Ext}^{p}(\mathcal{C}, \mathcal{G}) \rightarrow \operatorname{Ext}^{p}(\mathcal{B}, \mathcal{G}) \rightarrow \operatorname{Ext}^{p+1}(\mathcal{D}, \mathcal{G}) \rightarrow \ldots
$$

Analogously, the composition of $U$ with a $p$ extension of $\mathcal{D}$ by $\mathcal{F}$ gives the connecting homomorphisms in the long exact sequence

$$
\cdots \rightarrow \operatorname{Ext}^{p}(\mathcal{F}, \mathcal{B}) \rightarrow \operatorname{Ext}^{p}(\mathcal{F}, \mathcal{C}) \rightarrow \operatorname{Ext}^{p}(\mathcal{F}, \mathcal{D}) \rightarrow \operatorname{Ext}^{p+1}(\mathcal{F}, \mathcal{B}) \rightarrow \ldots
$$

Propositions 3.1 and 3.2 admit the following generalization.

Proposition 3.3. Let $\mathcal{B}$ be a sub-brane of $\mathcal{C}$ and $U$ the exact sequence (26).

1. The obstruction to an extension of the string $\mathbf{R} \in \operatorname{Ext}^{p}(\mathcal{B}, \mathcal{G})$ to a string stretching from $\mathcal{C}$ to $\mathcal{G}$ is the string

$$
\mathbf{R} \star U \in \operatorname{Ext}^{p+1}(\mathcal{C} / \mathcal{B}, \mathcal{G}) .
$$

2. The obstruction to a lift of the string $\mathbf{T} \in \operatorname{Ext}^{p}(\mathcal{F}, \mathcal{C} / \mathcal{B})$ to a string stretching from $\mathcal{F}$ to $\mathcal{C}$ is the string

$$
U \star \mathbf{T} \in \operatorname{Ext}^{p+1}(\mathcal{F}, \mathcal{B}) .
$$

\subsection{Vertex Operators and Local Extensions}

From now on in this subsection, we assume that $X$ is an $n$-dimensional algebraic variety and there exists a positive line bundle on $X$. So, each coherent sheaf $\mathcal{F}$ on $X$ admits a resolution consisting of locally free sheaves $[5,18]$

$$
0 \rightarrow \mathcal{E}_{n} \stackrel{\partial}{\rightarrow} \ldots \stackrel{\partial}{\rightarrow} \mathcal{E}_{1} \stackrel{\partial}{\rightarrow} \mathcal{E}_{0} \rightarrow \mathcal{F} \rightarrow 0
$$

Given $\mathcal{F}$ and $\mathcal{G}$ two coherent $\mathcal{O}$-modules, then the sheaf $\mathcal{E} x t^{k}(\mathcal{F}, \mathcal{G})$ is the cohomology object $h^{k}\left(\mathcal{H o m}\left(\mathcal{E}_{\bullet}, \mathcal{G}\right)\right)$ (by the proposition of [9] in page 234 above mentioned). We use these sheaves for determining the space $(13)$ of the vertex operators for an open string in the group $\operatorname{Ext}^{k}(\mathcal{F}, \mathcal{G})$. 
The exact sequence (36) gives rise to the short exact sequence

$$
0 \rightarrow \mathcal{K}_{p} \rightarrow \mathcal{E}_{p-1} \rightarrow \mathcal{K}_{p-1} \rightarrow 0,
$$

where $\mathcal{K}_{p}$ is the kernel of $\mathcal{E}_{p-1} \rightarrow \mathcal{E}_{p-2}$, or equivalently the cokernel of $\mathcal{E}_{p+1} \rightarrow \mathcal{E}_{p}$.

As $\mathcal{E} x t^{p}(\mathcal{F}, \mathcal{G})$ is the $p$-th cohomology of the complex $\mathcal{H o m}\left(\mathcal{E}_{\bullet}, \mathcal{G}\right)$, then $\mathcal{E}_{x} t^{p}(\mathcal{F}, \mathcal{G})$ is the sheaf associated to the presheaf

$$
U \mapsto h^{p}\left(\Gamma\left(U, \mathcal{H o m}\left(\mathcal{E}_{\bullet}, \mathcal{G}\right)\right)\right)
$$

where

$$
\Gamma\left(U, \mathcal{H o m}\left(\mathcal{E}_{\bullet}, \mathcal{G}\right)\right)=\operatorname{Hom}_{\left.\mathcal{O}\right|_{U}}\left(\mathcal{E}_{\bullet \mid U}, \mathcal{G}_{\mid U}\right) .
$$

Given a fine enough open covering $\mathfrak{U}=\left\{U_{\alpha}\right\}$ of $X$ and

$$
f \in H^{0}\left(X, \mathcal{E} x t^{p}(\mathcal{F}, \mathcal{G})\right),
$$

a global section of $\mathcal{E} x t^{p}(\mathcal{F}, \mathcal{G})$, they determine an element of

$$
h^{p}\left(\Gamma\left(U_{\alpha}, \mathcal{H o m}\left(\mathcal{E}_{\bullet}, \mathcal{G}\right)\right)\right),
$$

which in turn is the cohomology class of a cocycle

$$
f_{\alpha} \in \operatorname{Hom}_{\mathcal{O} \mid U_{\alpha}}\left(\mathcal{E}_{p \mid U_{\alpha}}, \mathcal{G}_{\mid U_{\alpha}}\right),
$$

i.e. satisfying $f_{\alpha} \circ \partial_{\mid U_{\alpha}}=0$. Hence, $f_{\alpha}$ admits a unique factorization through the cokernel of

$$
\partial_{\mid U_{\alpha}}: \mathcal{E}_{p+1 \mid U_{\alpha}} \rightarrow \mathcal{E}_{p \mid U_{\alpha}}
$$

In this way, $f_{\alpha}$ determines a unique morphism

$$
\widehat{f}_{\alpha}: \mathcal{K}_{p \mid U_{\alpha}} \rightarrow \mathcal{G}_{\mid U_{\alpha}}
$$

The pushout, i.e. the fibered coproduct, of the morphisms $\mathcal{K}_{p \mid U_{\alpha}} \rightarrow \mathcal{E}_{p-1 \mid U_{\alpha}}$ and $\widehat{f}_{\alpha}$ gives rise to the following commutative diagram in the category of $\mathcal{O}_{\mid U_{\alpha}}$-modules, where the short sequences are exact

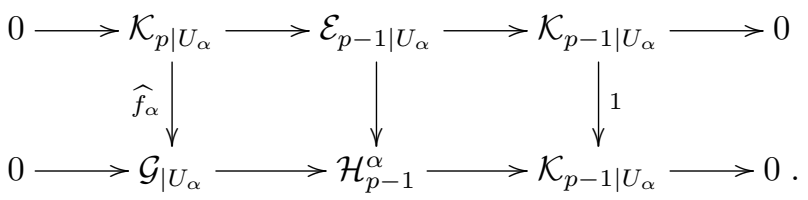

The sequence at the bottom in this diagram can be jointed with the exact sequence

$$
0 \rightarrow \mathcal{K}_{p-1} \rightarrow \mathcal{E}_{p-2} \rightarrow \mathcal{E}_{p-3} \rightarrow \cdots \rightarrow \mathcal{E}_{0} \rightarrow \mathcal{F} \rightarrow 0
$$

restricted to $U_{\alpha}$ and we obtain a $p$-extension of $\mathcal{G}_{\mid U_{\alpha}}$ by $\mathcal{F}_{\mid U_{\alpha}}$; i. e. the following exact sequence

$$
0 \rightarrow \mathcal{G}_{\mid U_{\alpha}} \rightarrow \mathcal{H}_{p-1}^{\alpha} \rightarrow \mathcal{E}_{p-2 \mid U_{\alpha}} \rightarrow \cdots \rightarrow \mathcal{E}_{0 \mid U_{\alpha}} \rightarrow \mathcal{F}_{\mid U_{\alpha}} \rightarrow 0
$$

If $U_{\alpha \beta}:=U_{\alpha} \cap U_{\beta} \neq \emptyset$, the obvious restrictions give rise to the following commutative diagram

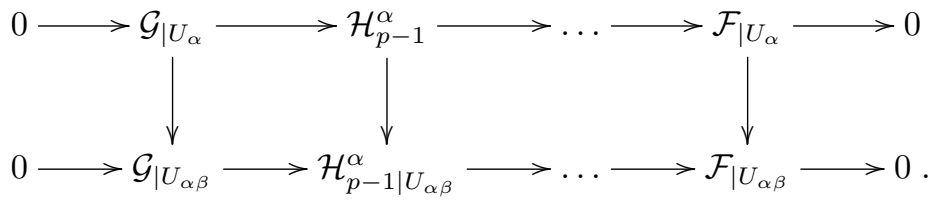

Since the restrictions of $\widehat{f}_{\alpha}$ and $\widehat{f}_{\beta}$ to $U_{\alpha \beta}$ patch together, one has isomorphisms

$$
\xi_{\beta \alpha}: \mathcal{H}_{p-1 \mid U_{\alpha \beta}}^{\alpha} \rightarrow \mathcal{H}_{p-1 \mid U_{\alpha \beta}}^{\beta} .
$$

In general, $\xi_{\alpha \beta} \circ \xi_{\beta \gamma} \neq \xi_{\alpha \gamma}$. Thus, the local extensions (40) do not define a global extension of $\mathcal{G}$ by $\mathcal{F}$. We can state the following theorem. 
Theorem 3.4. Let $\mathfrak{U}=\left\{U_{\alpha}\right\}$ be a sufficiently fine open covering of $X, \mathcal{F}$ and $\mathcal{G}$ coherent $\mathcal{O}$-modules. Each local operator of $H^{0}\left(X, \mathcal{E}_{x} t^{p}(\mathcal{F}, \mathcal{G})\right)$, with $p>0$ determines a p extension of $\mathcal{G}_{\mid U_{\alpha}}$ by $\mathcal{F}_{\mid U_{\alpha}}$, for all $\alpha$.

Remark 1. The local character of spaces $H^{0}\left(X, \mathcal{E} x t^{p}(\mathcal{F}, \mathcal{G})\right)$ appears clearer when they are compared with the Ext groups. For example, an element of $\operatorname{Ext}^{1}(\mathcal{F}, \mathcal{G})$ is given by a cocycle of the hypercohomology $\mathbb{H}^{1}\left(\mathfrak{U}, \mathcal{H o m}\left(\mathcal{E}_{\bullet}, \mathcal{G}\right)\right)$. This cocycle is a pair $\left(f=\left\{f_{\alpha}\right\}, h=\left\{h_{\alpha \beta}\right\}\right)$ with

$$
f_{\alpha} \in \Gamma\left(U_{\alpha}, \mathcal{H o m}\left(\mathcal{E}_{1}, \mathcal{G}\right)\right), \quad h_{\alpha \beta} \in \Gamma\left(U_{\alpha \beta}, \mathcal{H o m}\left(\mathcal{E}_{0}, \mathcal{G}\right)\right)
$$

satisfying

$$
\partial^{*} f=0, \quad \delta f=\partial^{*} h, \quad \delta h=0,
$$

where $\delta$ denotes the Čech coboundary operator and $\partial^{*}$ the one induced by $\partial$ on $\mathcal{H}$ om $\left(\mathcal{E}_{\bullet}, \mathcal{G}\right)$.

The condition $\partial^{*} f=0$ implies $f_{\alpha} \circ \partial_{\mid U_{\alpha}}=0$. Hence $f_{\alpha}$ factorizes through the cokernel of $\partial_{\mid U_{\alpha}}$. Thus, it is possible to carry out the preceding pushout construction and we obtain the diagram (39), with $p=1$. The condition $\delta f=\partial^{*}$ implies that the local extensions $\mathcal{H}_{0}^{\alpha}$ and $\mathcal{H}_{0}^{\beta}$ patch together on $U_{\alpha \beta}$. Now, from the condition $\delta h=0$, ones deduces the cocycle condition and the $\mathcal{H}_{0}^{\alpha}$ s define a global extension of $\mathcal{G}$ by $\mathcal{F}$. Essentially, this is the proof of the fact that $\operatorname{Ext}^{1}(\mathcal{F}, \mathcal{G})$ is the group of equivalence classes of length 1 extensions of $\mathcal{G}$ by $\mathcal{F}$.

The proof of Theorem 3.4 can extended directly to the vertex operators in $H^{q}\left(X, \mathcal{E} x t^{p}(\mathcal{F}, \mathcal{G})\right)$. Thus, we have the following theorem.

Theorem 3.5. Under the hypotheses of Theorem 3.4, each element of $H^{q}\left(X, \mathcal{E} x t^{p}(\mathcal{F}, \mathcal{G})\right)$, with $p>0$ determines a $p$ extension of $\mathcal{G}_{\mid U_{\alpha_{0} \ldots \alpha_{q}}}$ by $\mathcal{F}_{\mid U_{\alpha_{0} \ldots \alpha_{q}}}$, where

$$
U_{\alpha_{0} \ldots \alpha_{q}}=\bigcap_{j=0}^{q} U_{\alpha_{j}}
$$

In the case of strings between two $D 0$ branes the vertex operators define global extensions; in fact, they coincide with the respective strings. In the following example we consider this point.

Example. Let us assume that $\mathcal{I} \subset \mathcal{O}$ is a sheaf of regular ideals such that $Z$, the support of $\mathcal{O} / \mathcal{I}$, has dimension 0 . We will study the space of strings stretching between $\mathcal{O} / \mathcal{I}$ and itself. The set $Z$ can be regarded as a ringed space with structure sheaf $\mathcal{O}_{Z}=\mathcal{O} / \mathcal{I}$. A free resolution of $\mathcal{O}_{Z}$ is the Koszul complex, that we recall briefly (see $[6,8]$ for details).

If $e_{1}, \ldots, e_{n}$ is a set of symbols, we put $\mathcal{E}_{p}$ for the free $\mathcal{O}$-module generated by $\left\{e_{i_{1}} \wedge \cdots \wedge e_{i_{p}}\right\}$, with $i_{1}<\cdots<i_{p}$. That is, $\mathcal{E}_{p}$ is a free module with dimension $r:=\left(\begin{array}{l}n \\ p\end{array}\right)$.

The operator $\partial: \mathcal{E}_{p} \rightarrow \mathcal{E}_{p-1}$ is defined as follows: If $f_{1}, \ldots, f_{n}$ is a regular sequence of functions which generate the ideal $\mathcal{I}$ on an open $U$, then $\partial$ restricted to $U$ is defined by

$$
\partial\left(e_{i_{1}} \wedge \cdots \wedge e_{i_{p}}\right)=\sum_{j=1}^{p}(-1)^{j-1} f_{i_{j}} e_{i_{1}} \wedge \ldots \widehat{e}_{i_{j}} \cdots \wedge e_{i_{p}}
$$

The following exact sequence is a free resolution of $\mathcal{O}_{Z}$

$$
0 \rightarrow \mathcal{E}_{n} \stackrel{\partial}{\rightarrow} \mathcal{E}_{n-1} \stackrel{\partial}{\rightarrow} \ldots \stackrel{\partial}{\rightarrow} \mathcal{E}_{0}=\mathcal{O} \stackrel{\text { proj. }}{\rightarrow} \mathcal{O}_{Z} \rightarrow 0
$$

Applying the functor $\mathcal{H o m}\left(., \mathcal{O}_{Z}\right)$, one obtains the complex

$$
\left(\mathcal{H o m}\left(\mathcal{E}_{\bullet}, \mathcal{O}_{Z}\right), \partial^{*}\right)
$$

As the sheaf $\mathcal{E}_{p}$ is the direct $\operatorname{sum} \mathcal{O}^{\oplus r}$,

$$
\mathcal{H o m}\left(\mathcal{E}_{p}, \mathcal{O}_{Z}\right)=\left(\mathcal{O}_{Z}\right)^{\oplus r}
$$


Hence, the support of $\mathcal{H o m}\left(\mathcal{E}_{p}, \mathcal{O}_{Z}\right)$ is $Z$. On the other hand, since the $f_{j}$ vanish on $Z$, the coboundary $\partial^{*}$ operator induces the morphism zero in the stalk of $\mathcal{H o m}\left(\mathcal{E}_{p}, \mathcal{O}_{Z}\right)$ at any point of $Z$. Thus, the operator $\partial^{*}$ is identically zero.

The sheaves $\mathcal{E x t}^{p}\left(\mathcal{O}_{Z}, \mathcal{O}_{Z}\right)$ are the homology elements of the trivial complex (43). So,

$$
\mathcal{E} x t^{p}\left(\mathcal{O}_{Z}, \mathcal{O}_{Z}\right)=\mathcal{H o m}\left(\mathcal{E}_{p}, \mathcal{O}_{Z}\right)
$$

As $\mathcal{O}_{Z}$ is a skyscraper sheaf,

$$
H^{0}\left(X, \mathcal{O}_{Z}\right)=\bigoplus_{x \in Z}\left(\mathcal{O}_{Z}\right)_{x} \simeq \oplus_{x \in Z} \mathbb{C}
$$

and the other cohomology groups vanish. So, from (45) together with (44), it follows that

$$
\begin{aligned}
& H^{q}\left(X, \mathcal{E} x t^{p}\left(\mathcal{O}_{Z}, \mathcal{O}_{Z}\right)\right)=0, \quad \text { for } q \neq 0 . \\
& H^{0}\left(X, \mathcal{E} x t^{p}\left(\mathcal{O}_{Z}, \mathcal{O}_{Z}\right)\right) \simeq \oplus_{x \in Z} \mathbb{C}^{r}
\end{aligned}
$$

Therefore, in the second page of the Local-to-Global spectral sequence all the rows are identically zero unless one. Thus,

$$
\operatorname{Ext}^{p}\left(\mathcal{O}_{Z}, \mathcal{O}_{Z}\right)=H^{0}\left(X, \mathcal{E} x t^{p}\left(\mathcal{O}_{Z}, \mathcal{O}_{Z}\right)\right)
$$

That is, all the vertex operators for strings from the $D 0$ brane $\mathcal{O}_{Z}$ to itself are global extensions.

This result, for the case $p=1$, can be also deduced from the observations explained in Remark 1 . Let $\mathfrak{U}$ be a covering of $X$. Given $f \in H^{0}\left(X, \mathcal{E} x t^{1}\left(\mathcal{O}_{Z}, \mathcal{O}_{Z}\right)\right)$, it defines $f_{\alpha} \in \Gamma\left(U_{\alpha}, \mathcal{H o m}\left(\mathcal{E}_{1}, \mathcal{O}_{Z}\right)\right)$, as in (37). Assuming that each point of the discrete set $Z$ belongs to only one member of $\mathfrak{U}$, then the spaces $\Gamma\left(U_{\alpha \beta}, \mathcal{H o m}\left(\mathcal{E}_{0}, \mathcal{O}_{Z}\right)\right)$ and $\Gamma\left(U_{\alpha \beta}, \mathcal{H o m}\left(\mathcal{E}_{1}, \mathcal{O}_{Z}\right)\right)$ are zero, since $U_{\alpha \beta} \cap Z=\emptyset$. Hence taking $h=0 \in \Gamma\left(U_{\alpha \beta}, \mathcal{H o m}\left(\mathcal{E}_{0}, \mathcal{O}_{Z}\right)\right)$, the conditions (41) are trivially satisfied, and $f$ determines an element of $\operatorname{Ext}^{1}\left(\mathcal{O}_{Z}, \mathcal{O}_{Z}\right)$.

\section{Final Remarks}

We have applied methods and ideas from the cohomology of sheaves and from the theory of categories, for determining properties of the spaces of strings between general branes, i.e., objects in the derived category of coherent sheaves. We have also defined the vertex operators for those strings.

That generality had not been considered in the literature. The vertex operators usually considered are operators for strings stretching between the sheaves defined by the sections of vector bundles. Vertex operators for some Ext groups between non locally free sheaves are considered only exceptionally; for example, by studying branes on $X$ that are pushforward of holomorphic vector bundles on a submanifold of $X$ [12]. The branes usually taken into account are sheaves defined by sections of vector bundles on $X$ or vector bundles defined on subspaces of $X$, as the ones that arise from the $K$-homology of $X[3,11,17]$. Thus, we present applications of cohomological methods to the study of spaces of vertex operators that had not yet been considered.

When $X$ is a manifold acted by a Lie group $G$, it seems natural to define "equivariant" branes, and it is expected that the spaces of vertex operators for strings stretching between those branes support representations of $G$. In the particular case, when $X$ is a flag manifold of the group $G$ and the branes are locally free equivariant sheaves, perhaps the Borel-Weil-Bott theorem allows us to characterize the corresponding space of vertex operators. We think that the equivariance in the context of the branes is an issue worth exploring.

In this article, we used mathematical procedures and results in order to better understand concepts relative to the $B$-branes. In this context, the difficulty to derive the results consists in translating the physical concepts to a mathematical language and then the application of the appropriate mathematical tools. In general, these mathematical theories are far from the usual scientific background of physicists.

One ambitious different program would be to consider possible suggestions and insights for Mathematics that can be derived from methods used by studying the physics of the branes. This is an open tremendous challenge (see $[16])$. 
Acknowledgments. I thank E. Sharpe for his useful comments and the referee for his suggestions.

\section{Appendix}

In this section, we will prove Proposition 2.2 .

Let $K, L$ be complexes in an additive category $\mathfrak{A}$ and $f$ a morphism from $K$ to $L$. We denote by Con $(f)$ and $\mathrm{Cyl}(f)$ the corresponding mapping cone and mapping cylinder complexes. With a fixed a complex $F$, we put $\widehat{L}$ for denoting the complex of abelian groups $\operatorname{Hom}_{\mathfrak{A}}^{\bullet}(F, L)$, analogously $\widehat{K}=\operatorname{Hom}_{\mathfrak{A}}^{\bullet}(F, K)$ and $\widehat{f}:=\operatorname{Hom}_{\mathfrak{A}}^{\bullet}(F, f)$.

\section{Lemma 5.1.}

$$
\operatorname{Con}(\widehat{f})=\widehat{\operatorname{Con}(f)}, \quad \operatorname{Cyl}(\widehat{f})=\widehat{\operatorname{Cyl}(f)}
$$

Proof. By definition

$$
\widehat{L}^{m}=\prod_{i} \operatorname{Hom}_{\mathfrak{A}}\left(F^{i}, L^{m+i}\right),
$$

and coboundary operator

$$
d_{\widehat{L}}^{m}\left(s_{i}\right)=\left(d_{L}^{m+i} \circ s_{i}-(-1)^{m} s_{i+1} \circ d_{F}^{\hat{1}}\right),
$$

with $s_{i} \in \operatorname{Hom}_{\mathfrak{A}}\left(F^{i}, L^{m+i}\right)$. The complex $\widehat{K}$ and the operator $d_{\widehat{K}}$ are defined analogously.

As $f: K \rightarrow L$ is a morphism of complexes, it induces a complex morphism $\widehat{f}: \widehat{K} \rightarrow \widehat{L}$ by the formula

$$
\widehat{f}\left(r_{i}\right)=\left(f^{m+1+i} \circ r_{i}\right)
$$

with $r_{i} \in \operatorname{Hom}_{\mathfrak{A}}\left(F^{i}, K^{m+1+i}\right)$.

By definition,

$$
\operatorname{Con}(\widehat{f})^{m}=\widehat{K}^{m+1} \oplus \widehat{L}^{m}=\prod_{i}\left(\operatorname{Hom}_{\mathfrak{A}}\left(F^{i}, K^{m+1+i}\right) \oplus \operatorname{Hom}_{\mathfrak{A}}\left(F^{i}, L^{m+i}\right)\right) .
$$

The coboundary operator of this complex is defined by (see [10, page 23])

$$
d_{\mathrm{Con}(\widehat{f})}=\left(\begin{array}{cc}
-d_{\widehat{K}} & 0 \\
-\hat{f} & d_{\widehat{L}}
\end{array}\right)
$$

That is,

$$
d_{\mathrm{Con}(\widehat{f})}^{m}\left(\begin{array}{c}
r_{i} \\
s_{i}
\end{array}\right)=\left(\begin{array}{c}
-d_{K}^{m+1+i} \circ r_{i}-(-1)^{m} r_{i+1} \circ d_{F}^{i} \\
-f^{m+1+i} \circ r_{i}+d_{L}^{m+i} \circ s_{i}-(-1)^{m} s_{i+1} \circ d_{F}^{i}
\end{array}\right)
$$

On the other hand, we consider the complex

$$
\widehat{\operatorname{Con}(f)}=\operatorname{Hom}_{\mathfrak{A}}^{\bullet}(F, \operatorname{Con}(f)) .
$$

Hence,

$$
\widehat{\operatorname{Con}(f)}^{m}=\prod_{i} \operatorname{Hom}_{\mathfrak{A}}\left(F^{i}, \operatorname{Con}(f)^{m+i}\right)=\prod_{i} \operatorname{Hom}_{\mathfrak{A}}\left(F^{i}, K^{m+1+i} \oplus L^{m+i}\right) .
$$

From (48), it follows $\widehat{\operatorname{Con}(f)}^{m}=\operatorname{Con}(\widehat{f})^{m}$.

According to (46), the coboundary operator of $\widehat{\operatorname{Con}(f)}$ is defined by

$$
d_{\overline{\operatorname{Con}(f)}}^{m}\left(r_{i}, s_{i}\right)=d_{\operatorname{Con}(f)}^{m+i} \circ\left(r_{i}, s_{i}\right)-(-1)^{m}\left(r_{i+1}, s_{i+1}\right) \circ d_{F}^{i} .
$$

That is,

$$
d_{\overline{\operatorname{Con}(f)}}^{m}\left(\begin{array}{c}
r_{i} \\
s_{i}
\end{array}\right)=\left(\begin{array}{cc}
-d_{K} & 0 \\
-f & d_{L}
\end{array}\right)\left(\begin{array}{c}
r_{i} \\
s_{i}
\end{array}\right)-(-1)^{m}\left(\begin{array}{c}
r_{i+1} \\
s_{i+1}
\end{array}\right) \circ d_{F}^{i}
$$


This expression coincides with (50). So, we have the equality of the complexes

$$
\left.(\widehat{\operatorname{Con}(f}), d_{\widehat{\operatorname{Con}(f)}}\right)=\left(\operatorname{Con}(\widehat{f}), d_{\operatorname{Con}(\widehat{f})}\right) .
$$

The proof of the property relative to the cylinder is similar.

In general, if $l: \mathcal{M} \rightarrow \mathcal{N}$ is a morphism in the category $\mathfrak{C}$ of complexes of $\mathcal{O}$-modules and $U$ an open subset of $X$, then

$$
\operatorname{Con}\left(l_{\mid U}\right)=\mathcal{M}_{\mid U}[1] \oplus \mathcal{N}_{\mid U}=(\mathcal{M}[1] \oplus \mathcal{N})_{\mid U}=\operatorname{Con}(l)_{\mid U} .
$$

A similar relation holds for the mapping cylinder.

Let $\mathcal{P}, \mathcal{B}, \mathcal{A}$ objects of the category $\mathfrak{C}, g: \mathcal{B} \rightarrow \mathcal{A}$ a morphism in this category, and $U$ an open subset of $X$. Then $g_{\mid U}: \mathcal{B}_{\mid U} \rightarrow \mathcal{A}_{\mid U}$ is a morphism in the category of complexes of $\mathcal{O}_{\mid U}$-modules.

We put

$$
\widehat{g}=\mathcal{H o m}^{\bullet}(\mathcal{P}, g): \mathcal{H o m}^{\bullet}(\mathcal{P}, \mathcal{B}) \rightarrow \mathcal{H o m}^{\bullet}(\mathcal{P}, \mathcal{A})
$$

Analogously,

$$
\widehat{g_{\mid U}}:=\operatorname{Hom}_{\mathcal{O}_{\mid U}}^{\bullet}\left(\mathcal{P}_{\mid U}, g_{\mid U}\right): \operatorname{Hom}_{\mathcal{O}_{\mid U}}^{\bullet}\left(\mathcal{P}_{\mid U}, \mathcal{B}_{\mid U}\right) \rightarrow \operatorname{Hom}_{\mathcal{O}_{\mid U}}\left(\mathcal{P}_{\mid U}, \mathcal{A}_{\mid U}\right)
$$

\section{Lemma 5.2.}

$$
\operatorname{Con}(\widehat{g})(U)=\operatorname{Con}\left(\widehat{g_{\mid U}}\right), \operatorname{Cyl}(\widehat{g})(U)=\operatorname{Cyl}\left(\widehat{g_{\mid U}}\right) .
$$

Proof. By definition of the cone mapping and the $\mathcal{H}$ om functor,

$$
\begin{aligned}
\operatorname{Con}(\widehat{g})(U) & =\left(\mathcal{H o m}^{\bullet}(\mathcal{P}, \mathcal{B})[1] \oplus \mathcal{H o m}^{\bullet}(\mathcal{P}, \mathcal{A})\right)(U) \\
& =\operatorname{Hom}_{\mathcal{O}_{\mid U}}\left(\mathcal{P}_{\mid U}, \mathcal{B}_{\mid U}\right)[1] \oplus \operatorname{Hom}_{\mathcal{O}_{\mid U}}^{\bullet}\left(\mathcal{P}_{\mid U}, \mathcal{A}_{\mid U}\right)
\end{aligned}
$$

According (52), the last expression is precisely $\operatorname{Con}\left(\widehat{g_{\mid U}}\right)$.

Proof of Proposition 2.2. By definition of $\mathcal{H o m}$

$$
\mathcal{H o m}^{\bullet}(\mathcal{P}, \operatorname{Con}(g))(U)=\operatorname{Hom}_{\mathcal{O}_{\mid U}}\left(\mathcal{P}_{\mid U}, \operatorname{Con}(g)_{\mid U}\right)
$$

By (51)

$$
\operatorname{Hom}_{\mathcal{O}_{\mid U}}\left(\mathcal{P}_{\mid U}, \operatorname{Con}(g)_{\mid U}\right)=\operatorname{Hom}_{\mathcal{O}_{\mid U}}\left(\mathcal{P}_{\mid U}, \operatorname{Con}\left(g_{\mid U}\right)\right)
$$

Denoting $\mathfrak{A}$ the category of complex of $\mathcal{O}_{\mid U^{\text {-modules, }}}$ then $\operatorname{Hom}_{\mathcal{O}_{\mid U}}=\operatorname{Hom}_{\mathfrak{A}}$. With the notation used at the beginning of the Appendix, the right hand side of (54) is $\left.\widehat{\operatorname{Con}\left(g_{\mid}\right)}\right)$. From Lemmas 5.1 and 5.2, it follows

$$
\left.\widehat{\operatorname{Con}\left(g_{\mid U}\right.}\right)=\operatorname{Con}\left(\widehat{g_{\mid U}}\right)=\operatorname{Con}(\widehat{g})(U) \text {. }
$$

From (53), together with (54) and (55), we obtain

$$
\mathcal{H o m}^{\bullet}(\mathcal{P}, \operatorname{Con}(g))(U)=\operatorname{Con}(\widehat{g})(U)
$$

Similarly,

$$
\mathcal{H o m}^{\bullet}(\mathcal{P}, \operatorname{Cyl}(g))(U)=\operatorname{Cyl}(\widehat{g})(U) .
$$




\section{References}

1. Aspinwall, P. S.: D-branes on Calabi-Yau manifolds. In Progress in String Theory. Pages 1-152. World Sci. Publ. (2005).

2. Aspinwall, P. S. et al.: Dirichlet branes and mirror symmetry. Clay mathematics monographs vol 4. Amer. Math. Soc. (2009).

3. Baum, P.: $K$-homology and $D$-branes. In Superstrings, Geometry, Topology, and $C^{*}$-algebras. Vol 81, Proc. Sympos. Pure Math. 81-94. AMS (2009).

4. Bondal A., Kapranov M.: Representable functors, Serre functors, and mutations, Izv. Akad. Nauk SSSR, Ser.Mat., 53, 1183-1205 (1989); English transl. in Math. USSR Izv., 35, 519-541 (1990).

5. Fulton, W.: Intersection theory. Springer (1998).

6. Eisenbud, E.: Commutative algebra with a view toward algebraic geometry. Springer-Verlag (1995).

7. Gelfand, S. I., Manin, Y. I.: Methods of homological algebra. Springer (2003).

8. Griffiths, P., Harris, J.: Principles of algebraic geometry. John Wiley (1994).

9. Hartshorne R.: Algebraic geometry. Springer-Verlag (1983).

10. Iversen, B.: Cohomology of sheaves. Springer (1986).

11. Jia, B.: D-branes and $K$-homology. arXiv:1306.0535 [math. KT].

12. Katz, S., Sharpe, E.: D-branes, open string vertex operators, and Ext groups. Adv. Theor. Math. Phys. 6, 979-1030 (2003).

13. Kashiwara, M., Schapira, P.: Sheaves on manifolds. Springer-Verlag (2002).

14. Mac Lane, S.: Homology. Springer-Verlag (1975).

15. Mitchell, B.: Theory of categories. Academic Press. (1965).

16. Moore, G. W.: The impact of D-branes in Mathematics. (2014) (In www.physics.rutgers.edu/ gmoore).

17. Reis, R.M.G., Szabo, R.J.: Geometric K-homology of flat D-branes. Commun. Math. Phys. 266, 71-122 (2006).

18. Serre, J. P.: Faisceaux algébriques cohérents. Annals of Math. 61, 197-278 (1955).

19. Weibel, Ch. A.: An introduction to homological algebra. Cambridge U.P. (1997).

20. Witten, E.: Chern-Simmons gauge theory as a string theory. In H.Hofer et alt. editors The Floer memorial volume. 637-678. Birkhäuser (1995).

21. Witten, E.: Mirror manifolds and topological field theory. In Mirror Symmetry I. Edit S.-T. Yau. Pages 121-157. AMS (1998). 